\title{
Derrick Safety Monitoring System Based on Fiber Bragg Grating Strain Sensors
}

\author{
Yang $\mathrm{CHENG}^{*}$, Xianglin LU, Yuan GONG, Yu WU, and Yunjiang RAO \\ Key Laboratory of Optical Fiber Sensing \& Communications (Ministry of Education), University of Electronic Science \\ and Technology of China, Chengdu, 611731, China \\ *Corresponding author: Yang CHENGＥ-mail: ygong@uestc.edu.cn
}

\begin{abstract}
The fiber Bragg grating (FBG) strain sensor is used in the oil and gas derricks for the health monitoring. The system consisted of the FBG strain sensors, optical interrogator and monitoring software. 64 sensors were installed on a derrick for loading and unloading tests. The results showed that the monitoring system had the advantages of the high accuracy, good repeatability, large capacity and real-time monitoring ability.
\end{abstract}

Keywords: Fiber Bragg grating, Derrick, strain, monitor

Citation: Yang CHENG, Xianglin LU, Yuan GONG, Yu WU, and Yunjiang RAO, "Derrick Safety Monitoring System Based on Fiber Bragg Grating Strain Sensors," Photonic Sensors, DOI: 10.1007/s13320-013-0122-7.

\section{Introduction}

Derrick is an important part of the oil and gas field drilling rig. Its safety has great influences on the performance, reliability and lifetime of the whole rig. The derrick safety has been receiving much attention over the years. It is difficult for the conventional electrical strain sensors to monitor the strain of derrick components in the oil and gas field's harsh environment under high temperature, high pressure, chemical corrosion. The fiber Bragg grating (FBG) sensor is naturally safe and has the advantage of anti-electromagnetic interference, which is particularly important for the health monitoring in the field of oil and gas [1-8].

In our study, a derrick safety monitoring system based on the fiber Bragg grating strain sensors was developed and tested on a ZJ70DB derrick, and the test results showed that the monitoring system had good performance. It is especially suitable for oil and gas field derricks strain monitoring.

\section{Experiment and results}

\subsection{Principle}

When the FBG is strained, its central wavelength $\lambda_{B}$ drifts. The wavelength shift can be expressed as

$$
\Delta \lambda_{B}=\lambda_{B}\left(1-p_{e}\right) \varepsilon_{z} .
$$

where $p_{e}$ is the photo-elastic constant, and $\varepsilon_{z}$ is the strain [9]. The optical elastic constant of the typical quartz single-mode optical fiber was $p_{e}=0.22$. Generally, the strain sensitivity of FBG with no package was $1.2 \mathrm{pm} / \mu \varepsilon[10-11]$.

\subsection{FBG strain sensor}

The FBG strain sensor packaged with a steel structure is schematically shown in Fig. 1. The two ends of the FBG were fixed at Position 1 with ultraviolet glues. The packaged FBG sensor was mounted on the derrick via screws at Position 4. The strain of the derrick was transferred and converged at Position 5. Thus, the strain sensitivity of the FBG sensor was enhanced.

Received: 16 April 2013 / Revised version: 25 May 2013

(C) The Author(s) 2013. This article is published with open access at Springerlink.com 


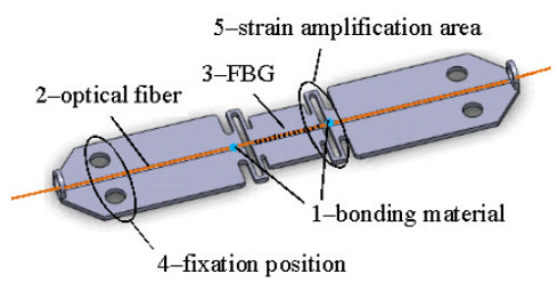

Fig. 1 Package structure of the FBG strain sensor.

Figure 2 shows the changes in the Bragg wavelength with the external displacement, which was applied to the sensor by fixing one end and stretching the other end of the packaged sensor. The linearity was 0.9996 . Then, the sensor was fixed together with an electrical strain sensor on a cantilever beam. Masses were added to the end of the beam, and the strain of the beam was measured simultaneously by the two kinds of sensors. The wavelength shifted as a function of the strain measured by the electrical sensor, as shown in Fig. 3 . It was indicated that both the repeatability and the linearity of the FBG strain sensor were good. The wavelength-strain sensitivity was statistically determined to be $0.0151 \pm 0.0002 \mathrm{~nm} / \mu \varepsilon$.

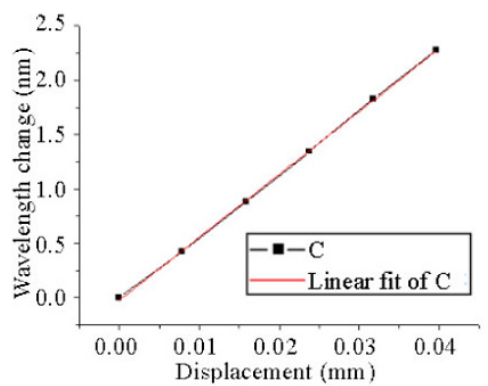

Fig. 2 Bragg wavelength changes with the external displacement.

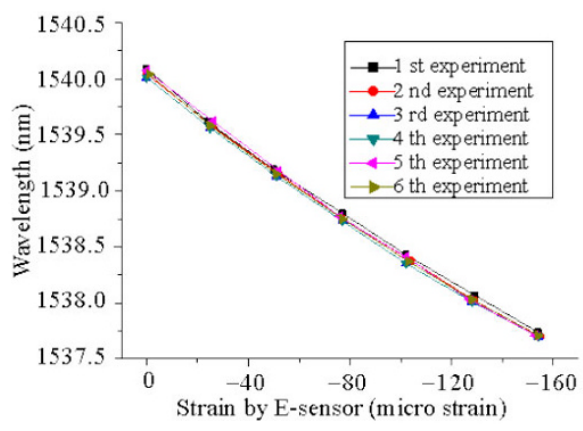

Fig. 3 Repeatability of the strain sensor.

\subsection{FBG strain sensing system}

The system structure is shown in Fig. 4. A Derrick security monitoring system was based on the optical fiber sensing network that consisted of 64 FBG strain sensors, which were installed on the derrick. The Bragg wavelength changed when the derrick deformed. The sensing optical cable also served as the transmission medium which resisted the electromagnetic interference and carried the sensing signal back to the interrogator. The wavelength shift was calculated to express the strain according to the calibrated coefficient. The entire data processing, monitoring and alarm task were completed by the software in the central processing host. The temperature fluctuations were measured by several FBG temperature sensors, and the influence on the FBG strain sensors was compensated to enhance the accuracy of the strain measurement.

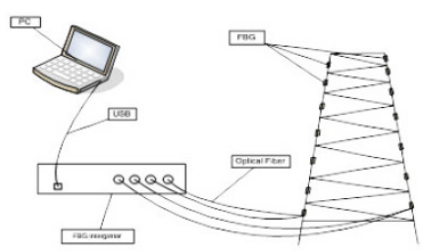

Fig. 4 Schematic of the derrick safety monitoring system

\subsection{Field test and results}

Totally, 64 FBG strain sensors were installed on a ZJ70DB derrick for the loading and unloading test. The photos of the field tests are shown in Fig. 5. A $1 \times 16$ optical switch was used in the FBG interrogator to expand the channel of it. The spectral range of the light source in the interrogator was about $40 \mathrm{~nm} .8$ channels were used and in each channel, and there were 8 FBG strain sensors fixed on the derrick in series. There were still 8 channels that could be used to add more sensing points. The number of sensors for each channel could be further extended by using the light source with the broader spectral range.

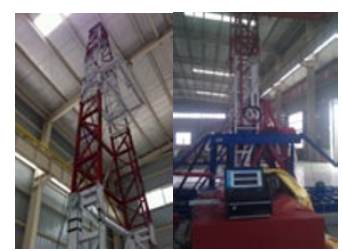

Fig. 5 Field test of the FBG strain sensing system. 


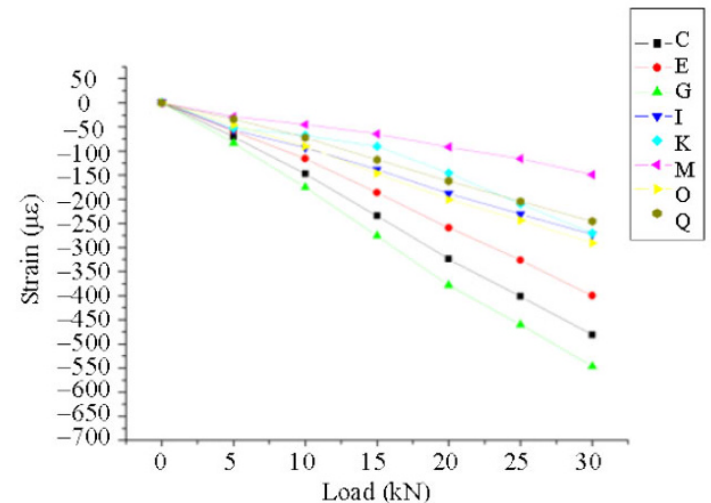

(a)

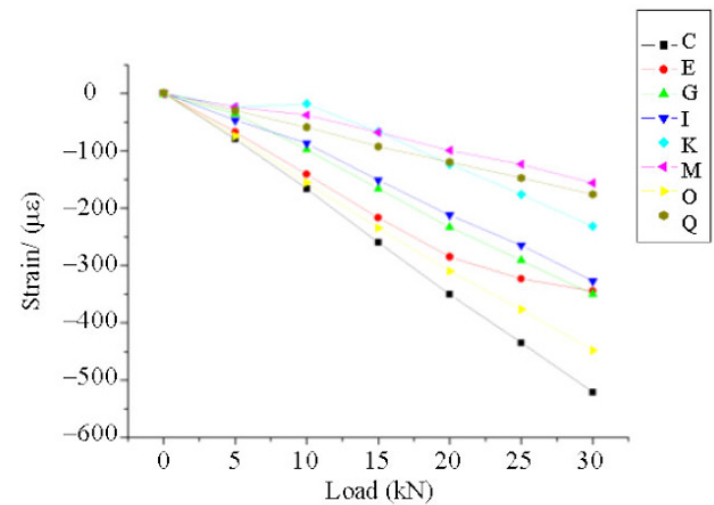

(c)

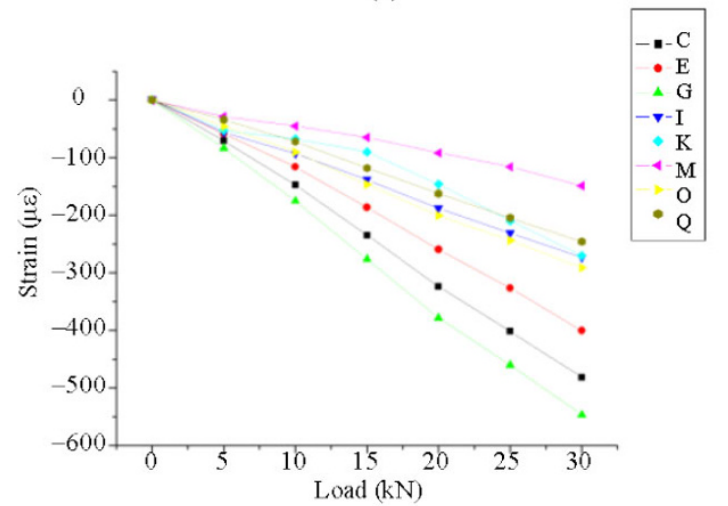

(e)

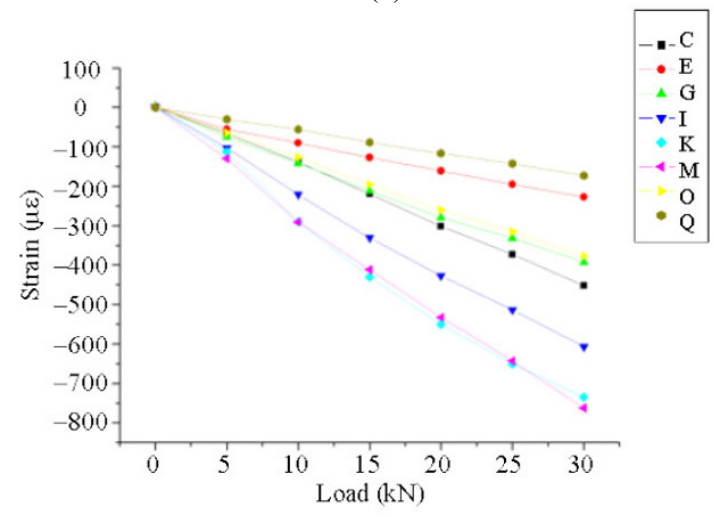

(g)

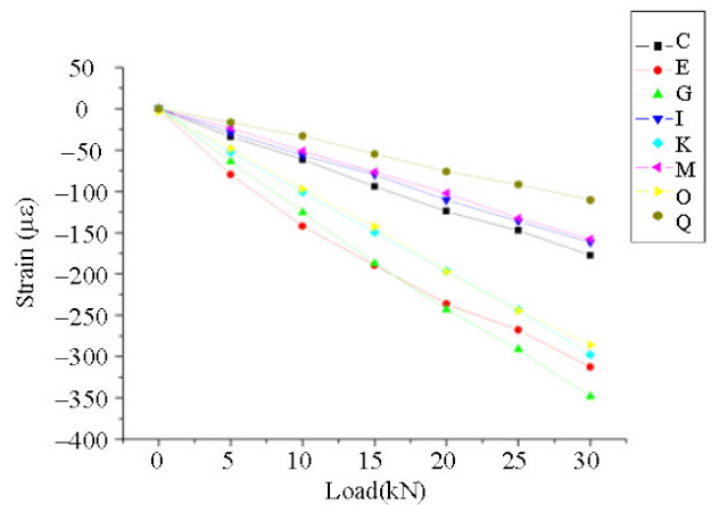

(b)

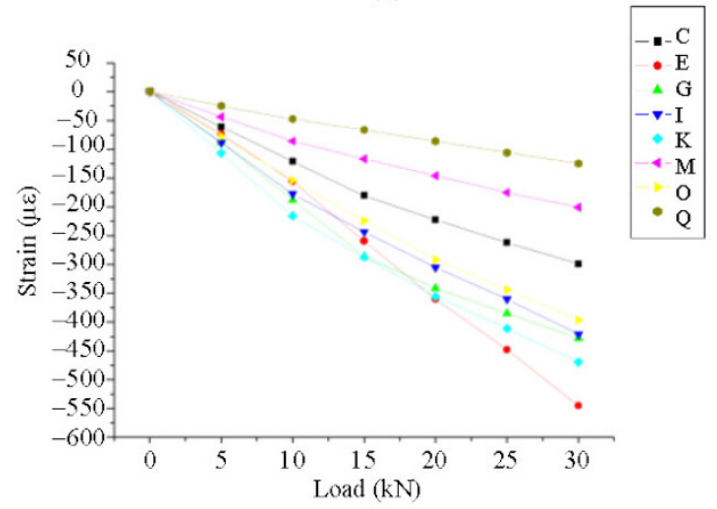

(d)

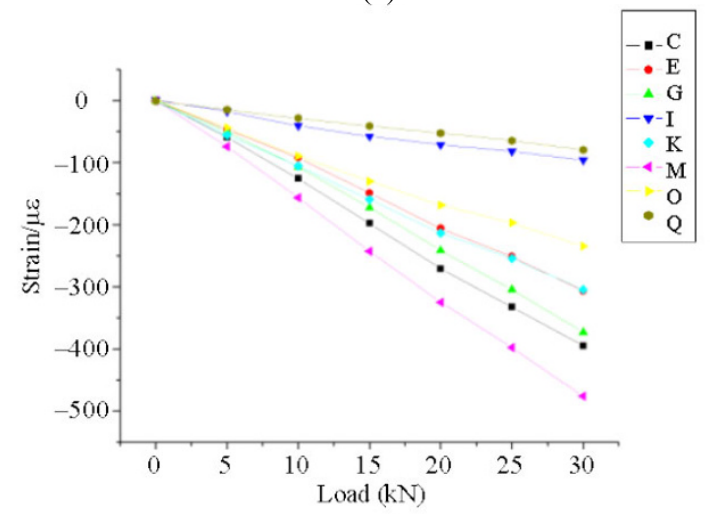

(f)

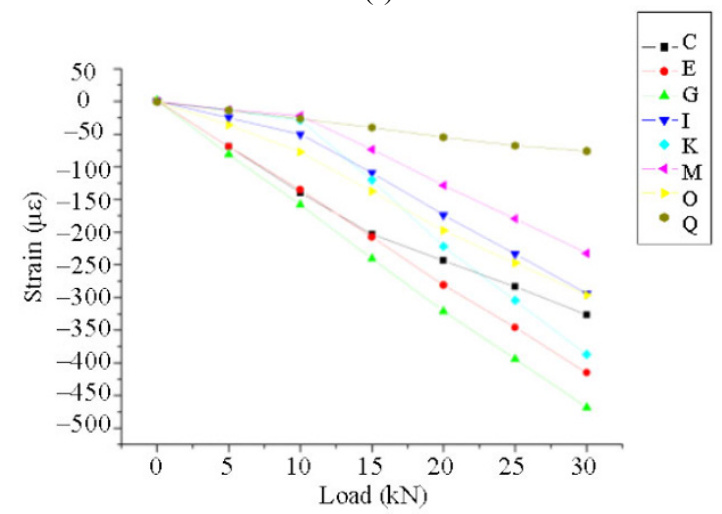

(h)

Fig. 6 Wavelength changes as a function of the applied load on the derrick: (a)-(h) show the field test results for channels $1-8$. 
The load of the derrick was added and then removed between 0 and $30 \mathrm{kN}$, in order to periodically change the applied strain on the derrick. The wavelength changes as a function of the load on the derrick are shown in Figs. 6(a)-6(h).

The derrick was then loaded from 0 to $50 \mathrm{kN}$ with a step of $10 \mathrm{kN}$, and the temporal sequence of the strain changes is shown in Fig. 7. The results showed that the monitoring system had high accuracy and good linearity. There was a recovery process of the measured strain, which indicated that the stiffness of the glue used to install the sensors onto the derrick needed to be enhanced.

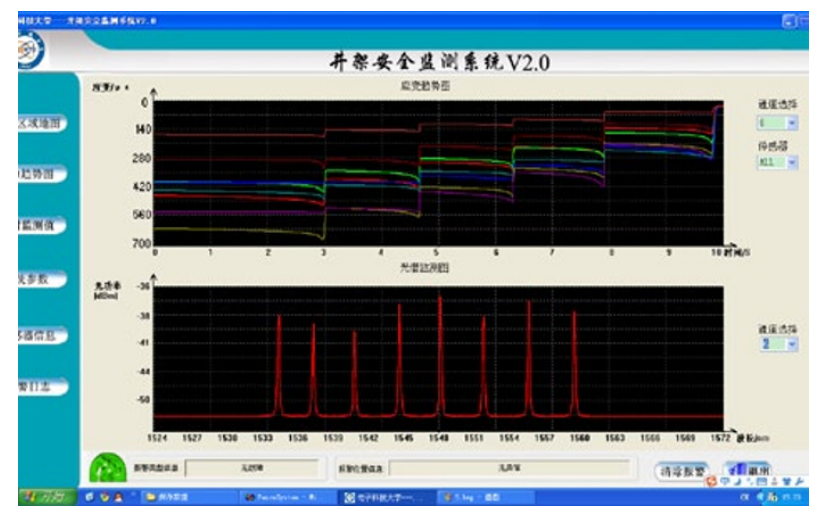

Fig. 7 Temporal sequence of the strain monitoring of the derrick.

\section{Conclusions}

A derrick safety monitoring system based on FBG strain sensors have been developed for the oil and gas field. The sensors were mounted on a derrick, and the performance of the system was tested. The results confirmed the feasibility of the field application.

Open Access This article is distributed under the terms of the Creative Commons Attribution License which permits any use, distribution, and reproduction in any medium, provided the original author(s) and source are credited.

\section{References}

[1] Y. J. Rao, "Recent progress in applications of in-fiber Bragg grating sensors," Optics and Lasers in Engineering, vol. 31, no. 4, pp. 297-324, 1999.

[2] A. D. Kersey, M. A. Davis, H. J. Patrick, M. LeBlanc, K. P. Koo, C. G. Askins, et al., "Fiber grating sensors," IEEE/OSA Journal of Lightwave Technology, vol. 15, no. 8, pp. 1442-1463, 1997.

[3] D. Jiang and W. He, "Review of applications for fiber Bragg grating sensors," Optoelectroncs and Laser, vol. 13, no. 4, pp. 420-430, 2002.

[4] G. C. Kahandawa, J. Epaarachchi, H. Wang, and K. T. Lau, "Use of FBG sensors for SHM in aerospace structures," Photonic Sensors, vol. 2, no. 3, pp. 203-214, 2012.

[5] M. Seaver, S. T. Trickey, and J. M. Nichols, "Strain measurements from FBGs embedded in rotating composite propeller blades," presented at 18th Conference on Optical Fiber Sensors, Cancun, Mexico, Oct. 23-27, 2006.

[6] Z. S. Wu and B. Xu, "A real-time structural parametric identification system based on fiber optic sensing and neural network algorithms," in Proc. SPIE, vol. 5047, pp. 252-262, 2003.

[7] S. D. Dyer, P. A. Williams, R. J. Espejo, J. D. Kofler, and S. M. Etzel, "Key metrology considerations for fiber Bragg grating sensors," in Proc. SPIE, vol. 5384, pp. 181-189, 2004.

[8] S. C. Kang, S. Y. Kim, S. B. Lee, S. W. Kwon, S. S. Choi, and B. Lee, "Temperature-independent strain sensor system using a titled fiber Bragg grating demodulator," IEEE Photonics Technology Letters, vol. 10, no. 10, pp. 1461-1463, 1998.

[9] Y. J. Rao, "In-fibre Bragg grating sensors," Measurement Science and Technology, vol. 8, no. 4, pp. 355-375, 1997.

[10] G. Meltz, W. W. Morey, W. H. Glenn, "Formation of Bragg gratings in optical fibers by a transverse holographic method," Optics Letters, vol. 14, no. 15, pp. 823-825, 1989.

[11] Y. J. Rao, A. B. Ribeiro, D. A. Jackson, L. Zhang, and I. Bennion, "Combined spatial- and time-division- multiplexing scheme for fibre grating sensors with drift-compensated phrase-sensitive detection," Optics Letters, vol. 20, no. 20, pp. 2149-2151, 1995. 